\title{
Almost everywhere well-posedness of continuity equations with measure initial data
}

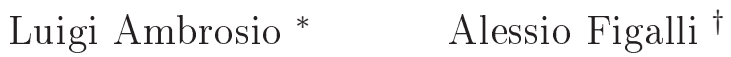

\begin{abstract}
The aim of this note is to present some new results concerning "almost everywhere" well-posedness and stability of continuity equations with measure initial data. The proofs of all such results can be found in [4], together with some application to the semiclassical limit of the Schrödinger equation.
\end{abstract}

\section{Resumé}

Dans cette note, nous présentons des nouveaux résultats concernant l'existence, l'unicité (au sens "presque partout") et la stabilité pour des équations de continuité avec données initiales mesures. Les preuves de tous ces résultats sont données dans [4], avec aussi des applications à la limite semiclassique pour l'équation de Schrödinger.

Starting from the seminal paper of DiPerna-Lions [7] (dealing mostly with the transport equation), in [1, 2] the well-posedness of the continuity equation

$$
\frac{d}{d t} \mu_{t}+\nabla \cdot\left(\boldsymbol{b}_{t} \mu_{t}\right)=0
$$

has been stronly related to well-posedness of the ODE (here we use the notation $\boldsymbol{b}(t, x)=\boldsymbol{b}_{t}(x)$ )

$$
\left\{\begin{array}{l}
\dot{\boldsymbol{X}}(t, x)=\boldsymbol{b}_{t}(\boldsymbol{X}(t, x)) \quad \text { for } \mathscr{L}^{1} \text {-a.e. } t \in(0, T) \\
\boldsymbol{X}(0, x)=x
\end{array}\right.
$$

for "almost every" $x \in \mathbb{R}^{d}$. More precisely, observe the concept of solution to (2) is not invariant under modification of $\boldsymbol{b}$ in Lebesgue negligible sets, while many applications of the theory to fluid dynamics (see for instance [11], [12]) and conservation laws need this invariance property. This leads to the concept of regular Lagrangian flow (RLF in short): one may ask that, for all $t \in[0, T]$, the image $\boldsymbol{X}(t, \cdot)_{\sharp} \mathscr{L}^{d}$ of the Lebesgue measure $\mathscr{L}^{d}$ under the flow map $x \mapsto \boldsymbol{X}(t, x)$ is still controlled by $\mathscr{L}^{d}$ (see Definition 1.1 below). Then, existence and uniqueness (up to $\mathscr{L}^{d}$-negligible sets) and stability of the $\operatorname{RLF} \boldsymbol{X}(t, x)$ in $\mathbb{R}^{d}$ hold true provided the functional version of (1), namely

$$
\frac{d}{d t} w_{t}+\nabla \cdot\left(\boldsymbol{b}_{t} w_{t}\right)=0
$$

is well-posed in the set of non-negative bounded integrable funtions $L_{+}^{\infty}\left([0, T] ; L^{1}\left(\mathbb{R}^{d}\right) \cap L^{\infty}\left(\mathbb{R}^{d}\right)\right)$.

Now, we may view (11) as an infinite-dimensional ODE in $\mathscr{P}\left(\mathbb{R}^{d}\right)$, the space of probability measures in $\mathbb{R}^{d}$ and try to obtain existence and uniqueness results for (1) in the same spirit of the finite-dimensional

\footnotetext{
*I.ambrosio@sns.it

$\dagger$ figalli@math.utexas.edu
} 
theory, starting from the simple observation that $t \mapsto \delta_{\boldsymbol{X}(t, x)}$ solves (11). We may expect that if we fix a "good" measure $\boldsymbol{\nu}$ in the space $\mathscr{P}\left(\mathbb{R}^{d}\right)$ of initial data, then existence, uniqueness $\boldsymbol{\nu}$-a.e. and stability hold. Moreover, for $\boldsymbol{\nu}$-a.e. $\mu$, the unique and stable solution of (1) starting from $\mu$ should be given by

$$
\boldsymbol{\mu}(t, \mu):=\int \delta_{\boldsymbol{X}(t, x)} d \mu(x) \quad \forall t \in[0, T], \mu \in \mathscr{P}\left(\mathbb{R}^{d}\right) .
$$

\section{Continuity equations and flows}

We use a standard and hopefully self-explainatory notation. Let $\boldsymbol{b}:[0, T] \times \mathbb{R}^{d} \rightarrow \mathbb{R}^{d}$ be a Borel vector field belonging to $L_{\text {loc }}^{1}\left([0, T] \times \mathbb{R}^{d}\right)$, and set $\boldsymbol{b}_{t}(\cdot):=\boldsymbol{b}(t, \cdot)$; we shall not work with the Lebesgue equivalence class of $\boldsymbol{b}$, although a posteriori the theory is independent of the choice of the representative.

Definition $1.1\left(\nu\right.$-RLF in $\left.\mathbb{R}^{d}\right)$. Let $\boldsymbol{X}(t, x):[0, T] \times \mathbb{R}^{d} \rightarrow \mathbb{R}^{d}$ and $\nu \in \mathscr{M}_{+}\left(\mathbb{R}^{d}\right)$ with $\nu \ll \mathscr{L}^{d}$ and with bounded density. We say that $\boldsymbol{X}(t, x)$ is a $\nu$-RLF in $\mathbb{R}^{d}$ (relative to $\boldsymbol{b}$ ) if the following two conditions are fulfilled:

(i) for $\nu$-a.e. $x$, the function $t \mapsto \boldsymbol{X}(t, x)$ is an absolutely continuous integral solution to the ODE (2) in $[0, T]$ with $\boldsymbol{X}(0, x)=x$;

(ii) $\boldsymbol{X}(t, \cdot)_{\sharp} \nu \leq C \mathscr{L}^{d}$ for all $t \in[0, T]$, for some constant $C$ independent of $t$.

By a simple application of Fubini's theorem this concept is, unlike the single condition (i), invariant in the Lebesgue equivalence class of $\boldsymbol{b}$. In this context, since all admissible initial measures $\nu$ are bounded above by $C \mathscr{L}^{d}$, uniqueness of the $\nu$-RLF can and will be understood in the following stronger sense: if $f, g \in L^{1}\left(\mathbb{R}^{d}\right) \cap L^{\infty}\left(\mathbb{R}^{d}\right)$ are nonnegative and $\boldsymbol{X}$ and $\boldsymbol{Y}$ are respectively a $f \mathscr{L}^{d}$-RLF and a $g \mathscr{L}^{d}$-RLF, then $\boldsymbol{X}(\cdot, x)=\boldsymbol{Y}(\cdot, x)$ for $\mathscr{L}^{d}$-a.e. $x \in\{f>0\} \cap\{g>0\}$.

Remark 1.2. We recall that the $\nu$-RLF exists for all $\nu \leq C \mathscr{L}^{d}$, and is unique, in the strong sense described above, under the following assumptions on $\boldsymbol{b}$ : $|\boldsymbol{b}|$ is uniformly bounded, $\boldsymbol{b}_{t} \in B V_{\mathrm{loc}}\left(\mathbb{R}^{d} ; \mathbb{R}^{d}\right)$ and $\nabla \cdot \boldsymbol{b}_{t}=g_{t} \mathscr{L}^{d} \ll \mathscr{L}^{d}$ for $\mathscr{L}^{1}$-a.e. $t \in(0, T)$, with

$$
\left\|g_{t}\right\|_{L^{\infty}\left(\mathbb{R}^{d}\right)} \in L^{1}(0, T), \quad\left|D \boldsymbol{b}_{t}\right|\left(B_{R}\right) \in L^{1}(0, T) \text { for all } R>0,
$$

where $\left|D \boldsymbol{b}_{t}\right|$ denotes the total variation of the distributional derivative of $\boldsymbol{b}_{t}$. (See [1] or [2] and the paper [6] for Hamiltonian vector fields.)

Given a nonnegative $\sigma$-finite measure $\boldsymbol{\nu} \in \mathscr{M}_{+}\left(\mathscr{P}\left(\mathbb{R}^{d}\right)\right)$, we denote by $\mathbb{E} \boldsymbol{\nu} \in \mathscr{M}_{+}\left(\mathbb{R}^{d}\right)$ its expectation, namely

$$
\int_{\mathbb{R}^{d}} \phi d \mathbb{E} \boldsymbol{\nu}=\int_{\mathscr{P}\left(\mathbb{R}^{d}\right)} \int_{\mathbb{R}^{d}} \phi d \mu d \boldsymbol{\nu}(\mu) \quad \text { for all } \phi \text { bounded Borel. }
$$

Definition 1.3 (Regular measures in $\left.\mathscr{M}_{+}\left(\mathscr{P}\left(\mathbb{R}^{d}\right)\right)\right)$. Let $\boldsymbol{\nu} \in \mathscr{M}_{+}\left(\mathscr{P}\left(\mathbb{R}^{d}\right)\right)$. We say that $\boldsymbol{\nu}$ is regular if $\mathbb{E} \boldsymbol{\nu} \leq C \mathscr{L}^{d}$ for some constant $C$.

Example 1.4. (1) The first standard example of a regular measure $\boldsymbol{\nu}$ is the law under $\rho \mathscr{L}^{d}$ of the map $x \mapsto \delta_{x}$, with $\rho \in L^{1}\left(\mathbb{R}^{d}\right) \cap L^{\infty}\left(\mathbb{R}^{d}\right)$ nonnegative. Actually, one can even consider the law under $\mathscr{L}^{d}$, and in this case $\boldsymbol{\nu}$ would be $\sigma$-finite instead of finite.

(2) If $d=2 n$ and $z=(x, p) \in \mathbb{R}^{n} \times \mathbb{R}^{n}$ (this factorization corresponds for instance to flows in a phase space), instead of considering the law of under $\rho \mathscr{L}^{2 n}$ of the map $(x, p) \mapsto \delta_{x} \otimes \delta_{p}$, one may also consider the law under $\rho \mathscr{L}^{n}$ of the map $x \mapsto \delta_{x} \times \gamma$, with $\rho \in L^{1}\left(\mathbb{R}_{x}^{n}\right) \cap L^{\infty}\left(\mathbb{R}_{x}^{n}\right)$ nonnegative and $\gamma \in \mathscr{P}\left(\mathbb{R}_{p}^{n}\right)$ bounded from above by a constant multiple of $\mathscr{L}^{n}$. 
We observe that Definition 1.1 has a natural (but not perfect) transposition to flows in $\mathscr{P}\left(\mathbb{R}^{d}\right)$ :

Definition 1.5 (Regular Lagrangian flow in $\mathscr{P}\left(\mathbb{R}^{d}\right)$ ). Let $\boldsymbol{\mu}:[0, T] \times \mathscr{P}\left(\mathbb{R}^{d}\right) \rightarrow \mathscr{P}\left(\mathbb{R}^{d}\right)$ and $\boldsymbol{\nu} \in$ $\mathscr{M}_{+}\left(\mathscr{P}\left(\mathbb{R}^{d}\right)\right.$ ). We say that $\boldsymbol{\mu}$ is a $\boldsymbol{\nu}-R L F$ in $\mathscr{P}\left(\mathbb{R}^{d}\right)$ (relative to $\boldsymbol{b}$ ) if

(i) for $\boldsymbol{\nu}$-a.e. $\mu,|\boldsymbol{b}| \in L_{\mathrm{loc}}^{1}\left((0, T) \times \mathbb{R}^{d} ; \mu_{t} d t\right), t \mapsto \mu_{t}:=\boldsymbol{\mu}(t, \mu)$ is continuous from $[0,1]$ to $\mathscr{P}\left(\mathbb{R}^{d}\right)$ with $\boldsymbol{\mu}(0, \mu)=\mu$ and $\mu_{t}$ solves (11) in the sense of distributions;

(ii) $\mathbb{E}\left(\boldsymbol{\mu}(t, \cdot)_{\sharp} \boldsymbol{\nu}\right) \leq C \mathscr{L}^{d}$ for all $t \in[0, T]$, for some constant $C$ independent of $t$.

Notice that condition (ii) is weaker than $\boldsymbol{\mu}(t, \cdot)_{\sharp} \boldsymbol{\nu} \leq C \boldsymbol{\nu}$ (which would be the analogue of (ii) in Definition 1.1 if we were allowed to choose $\nu=\mathscr{L}^{d}$ ), and it is actually sufficient and much more flexible for our purposes, since we would like to consider measures $\boldsymbol{\nu}$ generated as in Example 1.4(2).

\section{Existence, uniqueness and stability of the RLF}

In this section we recall the main existence and uniqueness results of the $\nu$-RLF in $\mathbb{R}^{d}$, and see their extensions to $\boldsymbol{\nu}$-RLF in $\mathscr{P}\left(\mathbb{R}^{d}\right)$. The following result is proved in [2, Theorem 19] for the part concerning existence and in [2, Theorem 16, Remark 17] for the part concerning uniqueness.

Theorem 2.1 (Existence and uniqueness of the $\nu$-RLF in $\mathbb{R}^{d}$ ). Assume that (3) has existence and uniqueness in $L_{+}^{\infty}\left([0, T] ; L^{1}\left(\mathbb{R}^{d}\right) \cap L^{\infty}\left(\mathbb{R}^{d}\right)\right)$. Then, for all $\nu \ll \mathscr{L}^{d}$ with bounded density the $\nu-R L F$ exists and is unique.

The next result shows that, uniqueness of (3) in $L_{+}^{\infty}\left([0, T] ; L^{1}\left(\mathbb{R}^{d}\right) \cap L^{\infty}\left(\mathbb{R}^{d}\right)\right)$ implies a stronger property, namely uniqueness of the $\boldsymbol{\nu}$-RLF.

Theorem 2.2 (Existence and uniqueness of the $\boldsymbol{\nu}$-RLF in $\mathscr{P}\left(\mathbb{R}^{d}\right)$ ). Assume that (3) has uniqueness in $L_{+}^{\infty}\left([0, T] ; L^{1}\left(\mathbb{R}^{d}\right) \cap L^{\infty}\left(\mathbb{R}^{d}\right)\right)$. Then, for all $\boldsymbol{\nu} \in \mathscr{M}_{+}\left(\mathscr{P}\left(\mathbb{R}^{d}\right)\right)$ regular, there exists at most one $\boldsymbol{\nu}-R L F$ in $\mathscr{P}\left(\mathbb{R}^{d}\right)$. If (3) has existence in $L_{+}^{\infty}\left([0, T] ; L^{1}\left(\mathbb{R}^{d}\right) \cap L^{\infty}\left(\mathbb{R}^{d}\right)\right)$, this unique flow is given by

$$
\boldsymbol{\mu}(t, \mu):=\int_{\mathbb{R}^{d}} \delta_{\boldsymbol{X}(t, x)} d \mu(x),
$$

where $\boldsymbol{X}(t, x)$ denotes the unique $\mathbb{E} \boldsymbol{\nu}-R L F$.

For the applications it is important to show that RLF's not only exist and are unique, but also that they are stable. In the statement of the stability result we shall consider measures $\boldsymbol{\nu}_{n} \in \mathscr{P}\left(\mathscr{P}\left(\mathbb{R}^{d}\right)\right)$, $n \geq 1$, and a limit measure $\boldsymbol{\nu}$. We shall assume that $\boldsymbol{\nu}_{n}=\left(i_{n}\right)_{\sharp} \mathbb{P}$, where $(W, \mathcal{F}, \mathbb{P})$ is a probability measure space and $i_{n}: W \rightarrow \mathscr{P}\left(\mathbb{R}^{d}\right)$ are measurable; we shall also assume that $\boldsymbol{\nu}=i_{\sharp} \mathbb{P}$, with $i_{n} \rightarrow i \mathbb{P}$-almost everywhere. (Recall that Skorokhod theorem (see [5, §8.5, Vol. II]) shows that weak convergence of $\boldsymbol{\nu}_{n}$ to $\boldsymbol{\nu}$ always implies this sort of representation, even with $W=[0,1]$ endowed with the standard measure structure, for suitable $i_{n}, i$.) The following formulation of the stability result is particularly suitable for the application to semiclassical limit of the Schrödinger equation.

Henceforth, we fix an autonomous vector field $\boldsymbol{b}: \mathbb{R}^{d} \rightarrow \mathbb{R}^{d}$ satisfying the following regularity conditions:

(a) $d=2 n$ and $\boldsymbol{b}(x, p)=(p, \boldsymbol{c}(x)),(x, p) \in \mathbb{R}^{d}, \boldsymbol{c}: \mathbb{R}^{n} \rightarrow \mathbb{R}^{n}$ Borel and locally integrable;

(b) there exists a closed $\mathscr{L}^{n}$-negligible set $S$ such that $c$ is locally bounded on $\mathbb{R}^{n} \backslash S$. 
Theorem 2.3 (Stability of the $\boldsymbol{\nu}$-RLF in $\mathscr{P}\left(\mathbb{R}^{d}\right)$ ). Let $i_{n}, i$ be as above and let $\boldsymbol{\mu}_{n}:[0, T] \times i_{n}(W) \rightarrow$ $\mathscr{P}\left(\mathbb{R}^{d}\right)$ be satisfying $\boldsymbol{\mu}_{n}\left(0, i_{n}(w)\right)=i_{n}(w)$ and the following conditions:

(i) (uniform regularity)

$$
\sup _{n \geq 1} \sup _{t \in[0, T]} \int_{W} \int_{\mathbb{R}^{d}} \phi d \boldsymbol{\mu}_{n}\left(t, i_{n}(w)\right) d \mathbb{P}(w) \leq C \int_{\mathbb{R}^{d}} \phi d x
$$

for all $\phi \in C_{c}\left(\mathbb{R}^{d}\right)$ nonnegative;

(ii) (uniform decay away from $S$ ) for some $\beta>1$

$$
\sup _{\delta>0} \limsup _{n \rightarrow \infty} \int_{W} \int_{0}^{T} \int_{B_{R}} \frac{1}{\operatorname{dist}^{\beta}(x, S)+\delta} d \boldsymbol{\mu}_{n}\left(t, i_{n}(w)\right) d t d \mathbb{P}(w)<\infty \quad \forall R>0 ;
$$

(iii) (space tightness) for all $\varepsilon>0, \mathbb{P}\left(\left\{w: \sup _{t \in[0, T]} \boldsymbol{\mu}_{n}\left(t, i_{n}(w)\right)\left(\mathbb{R}^{d} \backslash B_{R}\right)>\varepsilon\right\}\right) \rightarrow 0$ as $R \rightarrow \infty$;

(iv) (time tightness) for $\mathbb{P}$-a.e. $w \in W$, for all $n \geq 1$ and $\phi \in C_{c}^{\infty}\left(\mathbb{R}^{d}\right), t \mapsto \int_{\mathbb{R}^{d}} \phi d \boldsymbol{\mu}_{n}\left(t, i_{n}(w)\right)$ is absolutely continuous in $[0, T]$ and

$$
\lim _{M \uparrow \infty} \mathbb{P}\left(\left\{w \in W: \int_{0}^{T}\left|\left(\int_{\mathbb{R}^{d}} \phi d \boldsymbol{\mu}_{n}\left(t, i_{n}(w)\right)\right)^{\prime}\right| d t>M\right\}\right)=0 ;
$$

(v) (limit continuity equation)

$$
\lim _{n \rightarrow \infty} \int_{W}\left|\int_{0}^{T}\left[\varphi^{\prime}(t) \int_{\mathbb{R}^{d}} \phi d \boldsymbol{\mu}_{n}\left(t, i_{n}(w)\right)+\varphi(t) \int_{\mathbb{R}^{d}}\langle\boldsymbol{b}, \nabla \phi\rangle d \boldsymbol{\mu}_{n}\left(t, i_{n}(w)\right)\right] d t\right| d \mathbb{P}(w)=0
$$

for all $\phi \in C_{c}^{\infty}\left(\mathbb{R}^{d} \backslash\left(S \times \mathbb{R}^{n}\right)\right), \varphi \in C_{c}^{\infty}(0, T)$.

Assume, besides (a), (b) above, that (3) has uniqueness in $L_{+}^{\infty}\left([0, T] ; L^{1} \cap L^{\infty}\left(\mathbb{R}^{d}\right)\right)$. Then the $\boldsymbol{\nu}-R L F$ $\boldsymbol{\mu}(t, \mu)$ relative to $\boldsymbol{b}$ exists, is unique (by Theorem [2.2) and

$$
\lim _{n \rightarrow \infty} \int_{W} \sup _{t \in[0, T]} d \mathscr{P}\left(\boldsymbol{\mu}_{n}\left(t, i_{n}(w)\right), \boldsymbol{\mu}(t, i(w))\right) d \mathbb{P}(w)=0
$$

where $d_{\mathscr{P}}$ is any bounded distance in $\mathscr{P}\left(\mathbb{R}^{d}\right)$ inducing weak convergence of measures.

An example of application of the above stability result is the following: let $\alpha \in(0,1)$ and let $\psi_{x_{0}, p_{0}}^{\varepsilon}$ : $[0, T] \times \mathbb{R}^{n} \rightarrow \mathbb{C}$ be a family of solutions to the Schrödinger equation

$$
\left\{\begin{array}{l}
i \varepsilon \partial_{t} \psi_{x_{0}, p_{0}}^{\varepsilon}(t)=-\frac{\varepsilon^{2}}{2} \Delta \psi_{x_{0}, p_{0}}^{\varepsilon}(t)+U \psi_{x_{0}, p_{0}}^{\varepsilon}(t) \\
\psi_{x_{0}, p_{0}}^{\varepsilon}(0)=\varepsilon^{-n \alpha / 2} \phi_{0}\left(\frac{x-x_{0}}{\varepsilon^{\alpha}}\right) e^{i\left(x \cdot p_{0}\right) / \varepsilon}
\end{array}\right.
$$

with $\phi_{0} \in C_{c}^{2}\left(\mathbb{R}^{n}\right)$ and $\int\left|\phi_{0}\right|^{2} d x=1$. When the potential $U$ is of class $C^{2}$, it was proven in [9, 10, that for every $\left(x_{0}, p_{0}\right)$ the Wigner transforms $W_{\varepsilon} \psi_{x_{0}, p_{0}}^{\varepsilon}(t)$ converge, in the natural dual space $\mathcal{A}^{\prime}$ [10] for the Wigner transforms, to $\delta_{\boldsymbol{X}\left(t, x_{0}, p_{0}\right)}$ as $\varepsilon \downarrow 0$. Here $\boldsymbol{X}(t, x, p)$ if the unique flow in $\mathbb{R}^{2 n}$ associated to the Liouville equation

$$
\partial_{t} W+p \cdot \nabla_{x} W-\nabla U(x) \cdot \nabla_{p} W=0 .
$$


In [4], relying also on some a-priori estimates of [3] (see also [8]), the authors consider a potential $U$ which can be written as the sum of a repulsive Coulomb potential $U_{s}$ plus a bounded Lipschitz interation term $U_{b}$ with $\nabla U_{b} \in B V_{\text {loc }}$. We observe that in this case the equation (10) does not even make sense for measure initial data, as $\nabla U$ is not continuous. Still, they can prove full convergence as $\varepsilon \downarrow 0$, namely

$$
\lim _{\varepsilon \downarrow 0} \int_{\mathbb{R}^{d}} \rho\left(x_{0}, p_{0}\right) \sup _{t \in[-T, T]} d_{\mathcal{A}^{\prime}}\left(W_{\varepsilon} \psi_{x_{0}, p_{0}}^{\varepsilon}(t), \delta_{\boldsymbol{X}\left(t, x_{0}, p_{0}\right)}\right) d x_{0} d p_{0}=0 \quad \forall T>0
$$

for all $\rho \in L^{1}\left(\mathbb{R}^{2 n}\right) \cap L^{\infty}\left(\mathbb{R}^{2 n}\right)$ nonnegative, where $\boldsymbol{X}(t, x, p)$ if the unique $\mathscr{L}^{2 n}$-RLF associated to (10) and $d_{\mathcal{A}^{\prime}}$ is a bounded distance inducing the weak* topology in the unit ball of $\mathcal{A}^{\prime}$.

The proof of (11) relies on an application of Theorem 2.3 to the Husimi transforms of $\psi_{x_{0}, p_{0}}^{\varepsilon}(t)$. The scheme is sufficiently flexible to allow more general families of initial conditions displaying partial concentration, of position or momentum, or no concentration at all: for instance, the limiting case $\alpha=1$ in (9) (related to Example 1.4(2)) leads to

$$
\lim _{\varepsilon \downarrow 0} \int_{\mathbb{R}^{d}} \rho\left(x_{0}\right) \sup _{t \in[-T, T]} d_{\mathcal{A}^{\prime}}\left(W_{\varepsilon} \psi_{x_{0}, p_{0}}^{\varepsilon}(t), \boldsymbol{\mu}\left(t, \mu\left(x_{0}, p_{0}\right)\right)\right) d x_{0}=0 \quad \forall p_{0} \in \mathbb{R}^{n}, T>0
$$

for all $\rho \in L^{1}\left(\mathbb{R}^{n}\right) \cap L^{\infty}\left(\mathbb{R}^{n}\right)$ nonnegative, with $\boldsymbol{\mu}(t, \mu)$ given by (4) and $\mu\left(x_{0}, p_{0}\right)=\delta_{x_{0}} \times\left|\hat{\phi}_{0}\right|^{2}\left(\cdot-p_{0}\right) \mathscr{L}^{n}$.

\section{References}

[1] L.Ambrosio: Transport equation and Cauchy problem for BV vector fields. Invent. Math., 158 (2004), 227-260.

[2] L.Ambrosio: Transport equation and Cauchy problem for non-smooth vector fields. Lecture Notes in Mathematics "Calculus of Variations and Non-Linear Partial Differential Equations" (CIME Series, Cetraro, 2005) 1927, B. Dacorogna, P. Marcellini eds., 2-41, 2008.

[3] L.Ambrosio, G.Friesecke, J.Giannoulis: Passage from quantum to classical molecular dynamics in the presence of Coulomb interactions. Submitted paper, 2009.

[4] L.Ambrosio, A.Figalli, G.Friesecke, J.Giannoulis: Well posedness of transport equations with measure initial data and convergence of Wigner measures. Work in progress.

[5] V.Bogachev: Measure Theory, Voll. I and II. Springer, 2007.

[6] F.Bouchut: Renormalized solutions to the Vlasov equation with coefficients of bounded variation. Arch. Ration. Mech. Anal., 157 (2001), 75-90.

[7] R.J.DiPerna, P.L.Lions: Ordinary differential equations, transport theory and Sobolev spaces. Invent. Math., 98 (1989), 511-547.

[8] A.Figalli, T.Paul: work in progress.

[9] P.GÉrard: Mesures semi-classiques et ondes de Bloch. Seminaire sur les Équations aux Dérivées Partielles, 1990-1991. Exp. No. XVI, 19 pp., École Polytechnique, Palaiseau, 1991.

[10] P.L.Lions, T.Paul: Sur les mesures de Wigner. Rev. Mat. Iberoamericana, 9 (1993), 553-618.

[11] P.L.Lions: Mathematical topics in fluid mechanics, Vol. I: incompressible models. Oxford Lecture Series in Mathematics and its applications, 3 (1996), Oxford University Press.

[12] P.L.Lions: Mathematical topics in fluid mechanics, Vol. II: compressible models. Oxford Lecture Series in Mathematics and its applications, 10 (1998), Oxford University Press. 